\title{
REVIEWS
}

\section{Multiple organ failure. How valid is the "two hit" model?}

\author{
Roger Saadia, Moshe Schein
}

\begin{abstract}
Inflammatory "one hit" and "two hit" models have recently been proposed to account for the development of multiple organ failure (MOF) in trauma and critically ill surgical patients when no source of infection can be found. In the "one hit" model, the initial insult is so massive that a systemic inflammatory response syndrome is triggered and leads rapidly to MOF. In the "two hit" scenario, initially less severely injured patients eventually develop MOF as a result of a reactivation of their inflammatory response caused by an adverse and often minor intercurrent event. At first sight, the theory is attractive because it seems to fit commonly observed clinical patterns. Indeed, injured patients often respond to initial resuscitation but, after an insult of some sort, develop organ dysfunction and die. The "two hit" model is furthermore mirrored at the cellular level. Inflammatory cells are indeed susceptible of being primed by an initial stimulus and reactivated subsequently by a relatively innocuous insult. However, in the absence of clinical and biological corroboration based on cytokine secretion patterns, these models should not be accepted uncritically.

( Accid Emerg Med 1999;16:163-167)
\end{abstract}

Surgery, Baragwanath

Hospital and the

University of the

Witwatersrand,

Johannesburg, South

Africa

R Saadia

Department of

Surgery, New York

Methodist Hospital

and Cornell University

Medical College, New

York, USA

M Schein

Correspondence to: Professor R Saadia, Department of Surgery, Medical School, 7 York

Road, Parktown, 2193

Johannesburg, South Africa

(e-mail: roger.s@pixie.co.za).

Accepted 9 January 1999

Keywords: multiple organ failure; two hit model; post-trauma multiple organ failure

The recognition of multiple organ failure (MOF) as a syndrome goes back to the $1970 \mathrm{~s}^{1-3}$ It was at first well accepted that, in surgical and trauma patients, a focus of infection (usually an occult intra-abdominal abscess) was the most common trigger for MOF. ${ }^{4}$ The view has finally prevailed however under the weight of accumulated clinical evidence and current experience that, more often than not, no such infectious focus can be found in patients developing $\mathrm{MOF}^{56}$ And the observation that drainage of an intraabdominal abscess does not necessarily reverse MOF has added more urgency to the question of how MOF gets triggered and perpetuated in the absence of infection. ${ }^{7}$ In this regard, intes- tinal bacterial translocation (the "gut hypothesis") generated, for a while, keen interest on the basis of compelling animal experimental evidence, ${ }^{8}$ but could not be vindicated by clinical studies. ${ }^{9}$ At the same time, semantic progress has taken place. The clinical response resulting from a non-specific insult (and the precursor of MOF) has been termed "systemic inflammatory response syndrome" (SIRS), and "sepsis" has been defined as SIRS caused by infection, ${ }^{10}$ providing a more precise conceptual framework for investigating the pathogenesis of MOF

In the 1990s, an "inflammatory" theory has been proposed to explain the occurrence of MOF without infection ${ }^{11-13}$ : a "one hit model" would account for early MOF after massive trauma, while a "two hit model" would describe how initially less severely injured patients become susceptible to an amplified SIRS response, leading eventually to MOF. Partly because of the lack of competing theories and partly because, at least superficially, they seem to fit the clinical facts, these models have steadily gained popularity not only in trauma but also in general surgical and intensive care circles. The purpose of this paper is to give an account of these models. Particular attention is paid to the two hit hypothesis: both its clinical and biological foundations are examined and its validity assessed in the light of available clinical evidence.
"One hit" and "two hit" models: clinical and biological foundations

Most trauma patients develop a clinical response, SIRS, which seems to be beneficial and recover. A minority of patients, however, develop MOF. The theory holds, in brief, that there are two patterns of MOF, "early" (within 72 hours of the injury) and "delayed".${ }^{13}$ In the one hit model, the initial injury is so massive that a severe SIRS is precipitated, resulting in early and often lethal MOF, usually independent of infection. In the alternative scenario, the two hit model (referred to also as the "multiple hit" or "sequential hit" model), a less severe initial surgical or traumatic insult (first hit) is responsible for a moderate state of SIRS; subsequent insults (second and sequential hits), infectious or non-infectious, may amplify the pre-existing inflammatory state into an exag- 
gerated SIRS sufficient to induce delayed MOF. The initial event is said to prime the host in a way such that an otherwise innocuous secondary insult (the activating event) unleashes an uncontrolled inflammatory response and MOF. The two hit model in particular owes its appeal to the observation that severely ill or injured patients are commonly subjected to sequential insults that seem to contribute, singly or cumulatively, to their unfavourable outcome.

It is important to note that the sequential hits are described as "otherwise innocuous inflammatory insults", ${ }^{13}$ in other words insults of a magnitude such that they would not be attended by a significant morbidity should they occur in an otherwise healthy host. But the theory remains remarkably vague about what precisely could count as activating events: perhaps translocational gut endotoxaemia (and no hard evidence is offered in support of this), ${ }^{11}{ }^{12}$ or "virtually any insult, including infection, anaesthesia, an episode of hypotension, etc". ${ }^{14}$

The description of post-injury MOF as a syndrome occurring in two different temporal patterns (early or late) and the more common association of infection with the late form of MOF is supported, in the trauma literature, by a few clinical studies. Faist et al noted that $44 \%$ of their post-injury MOF patients developed organ failure within 12 to 36 hours while, in the remaining $56 \%$, MOF set in late (seven to eight days). The latter group was uniformly associated with sepsis. ${ }^{5}$ In another German series, two similar peaks of organ failure were observed. In $50 \%$ of the late MOF patients, infections were chronologically related to the onset of organ failure. ${ }^{15}$ In two additional studies by the Denver group, an identical bimodal distribution of MOF onset was noted, but the exclusive association of infection with late MOF was less clear cut. ${ }^{1617}$

In the two hit hypothesis, the initial insult primes the host as a first step. An activating event subsequently triggers a generalised autodestructive inflammatory response followed by MOF. This sequence is the attractive correlate of a cellular phenomenon that lends the model biological credibility. Indeed, the succession of priming and activation of certain cell lines allows mildly injurious stimuli to synergistically set off the inflammatory machinery and cause tissue damage. Low doses of endotoxin, chemotactic agents, various other mediators, and cytokines prime inflammatory cells like polymorphonuclear neutrophils, macrophages, and endothelial cells in a manner such that a subsequent otherwise relatively innocuous stimulus induces cytokine mediated tissue injury that histologically and functionally resembles MOF. ${ }^{18-21}$ In a rabbit model, haemorrhage caused an increased susceptibility of the cardiovascular and pulmonary systems to the adverse effect of endotoxin administered in an otherwise harmless dose. The authors suggested that part of the interaction between haemorrhagic shock and endotoxin administration may be the result of macrophage stimulation leading to cytokine production. ${ }^{22}$ The Denver group have developed a rodent model to study neutrophil priming and reactivation. In a series of experiments, they were able to demonstrate that superior mesenteric artery clamping (analogous to shock induced splanchnic hypoperfusion) induces an ischaemia-reperfusion phenomenon capable of priming neutrophils in the mesenteric bed. These neutrophils enter subsequently the systemic circulation and gain access to the pulmonary vascular system, where they are susceptible to reactivation. At this stage, the administration of a small dose of endotoxin unleashes their cytotoxic effects. ${ }^{23} \mathrm{~A}$ comprehensive review of cellular priming and activation was published recently. ${ }^{24}$

\section{Evaluation of the two hit model}

It seems firstly that the clinical appeal of the two hit model stems from the common observation that, in the surgical intensive care unit, critically ill patients are subjected to a variety of insults that are responsible for their deteriorating organ function (and sometimes fatal outcome). As mentioned above, the formulation of the theory falls short in defining precisely what these aggravating events might be. Inadequate resuscitation in the pre-hospital setting or the emergency room with prolonged hypotension or untreated pain may act as such a trigger. Several other possibilities, occurring later in the clinical course, come to mind: a re-look laparotomy, delayed fracture fixation in a polytrauma patient, the performance of a tracheostomy, an intercurrent infection (pneumonia, catheter related septicaemia), a complication of invasive monitoring, or the transfer from the intensive care unit to the radiology suite of an unstable patient. Although these insults are often referred to by clinicians as second hits, it is to be noted that not all of them can be described as "otherwise innocuous", as the theory would have it. Furthermore, while there is no denying that any one of these events may be responsible for the deterioration of critically ill surgical patients, it remains to be proved that this indeed is the result of the amplification of their pre-existing inflammatory state. At the very least, the secondary events ought to perpetuate the initial inflammatory response over a period of time sufficiently long to cause tissue injury. It is necessary, in other words, to provide evidence that these patients are in a primed state and that the subsequent incidents are genuine activating events, "fuelling the inflammatory fire", rather than mere complications compounding an already grave clinical state. This is a prerequisite for accepting the two hit model as an accurate description of the clinical facts.

Tissue injury through cellular priming and activation is primarily mediated by cytokines. There has been rapid accumulation of data implicating these mediators in the pathophysiology of SIRS..$^{25}$ Four cytokines in particular have been incriminated: tumour necrosis factor- $\alpha$ (TNF- $\alpha$ ), interleukin-1 $\beta$ (IL-1 $\beta$ ), interleukin-6 (IL-6), and interleukin-8 (IL-8). The cytokine cascade is initiated when a stimulus such as Gram negative bacterial endotoxin induces the production and release 
of "proximal" cytokines, TNF- $\alpha$ and IL-1 $\beta$. These are responsible for most of the pathophysiological disturbances characteristic of sepsis. Endotoxin and the proximal cytokines stimulate in turn the production of "distal" cytokines, such as IL-6 and IL-8, which seem to intensify and perpetuate the inflammatory state. Therefore, the pattern of cytokine production between the initial injury and the development, in some of the patients, of MOF could provide the means of evaluating the one hit and two hit models.

Cytokine production after surgery or trauma was documented in several studies. ${ }^{26-33}$ The results lack the consistency required for a totally homogeneous picture. Although increased early post-injury concentrations of TNF- $\alpha$, IL- $1 \beta$, and IL- 6 have been recorded in most of these studies, the correlation between the early high cytokine concentrations and the development of MOF is, however, not sufficiently strong to promote these cytokines to the status of MOF predictors. None of these studies have concentrated on early MOF, and it is therefore impossible to test the one hit model. Furthermore, the influence of adverse clinical events on cytokine production in the interval between initial insult and MOF was not assessed; certainly no secondary peaks were recorded in that interval.

Two recent studies examined specifically the role of subsequent surgical procedures as secondary inflammatory insults in the development of late MOF. In a series of 106 trauma patients requiring reoperations (facial reconstruction, pelvic or long bone osteosynthesis, tracheostomy, and others), it was found that preoperative concentrations of neutrophil elastase, $\mathrm{C}$ reactive protein, and platelet count were good predictors of subsequent $\mathrm{MOF}^{34}$ Despite the authors' claim that "secondary operations ... may act as a second-hit in severely injured patients and trigger postoperative organ failure", the study merely identified preoperative markers of MOF. Support for the second hit phenomenon would have required the demonstration of an escalating (or at least sustained) inflammatory response after the secondary intervention and leading to MOF. In another study, the effect of re-look laparotomies on the cytokine profile of 15 non-trauma patients with severe peritonitis was investigated. ${ }^{35}$ There was a sharp increase of IL-6 concentrations after reoperation, with unchanged concentrations of endotoxin and TNF- $\alpha$. While initial IL- 6 concentrations were shown in previous studies to be markers of $M O F,{ }^{33}$ no correlation was sought in this study between IL-6 and patient outcome. And it remains uncertain whether the postreoperative IL-6 peak was a marker of unfavourable prognosis or a mere epiphenomenon.

\section{Can the two hit model be salvaged?}

Despite the hitherto lack of corroborating evidence, it is still premature to discard the two hit hypothesis. Good clinical studies investigating the influence of adverse events on cytokine production in the critically ill surgical patient are still awaited. Such studies may have to overcome two potential sets of difficulties. Firstly, they should take into account the timing of the second hit in relation to the primary insult, as the susceptibility for reactivation of the inflammatory machinery in a primed host may be restricted to a relatively narrow time window. Secondly, concentrations of circulatory cytokines may provide a distorted reflection of the magnitude of the inflammatory response. It has been suggested recently that the cytokine response is compartmentalised $^{36}$ : local, intratissular cytokines exerting their effect in a paracrine fashion may have greater biological relevance than their concomitant plasma spillover.

The phenomenon of cytokine compartmentalisation has been documented in several studies. In experimental pancreatitis, portal vein concentrations of TNF- $\alpha$ were higher than those measured in the hepatic vein. ${ }^{37}$ After colectomy, IL-6 was higher in the portal vein than in the systemic circulation. ${ }^{38}$ They were also higher in burned skin than in the blood. ${ }^{39}$ Bacterial peritonitis is characterised by a powerful compartmentalised intraperitoneal cytokine response with plasma cytokine concentrations representing only the tip of the iceberg. ${ }^{3640}$ Cytokine and other mediators were assayed in the plasma and peritoneal exudate of patients undergoing electively staged relaparotomies for severe intra-abdominal infection. ${ }^{40}$ Peritoneal concentrations were many times higher than those measured simultaneously in the plasma. The magnitude of the mediator response did correlate with outcome (higher concentrations tended to persist in non-survivors), but the contribution of reoperative trauma to this response remained unclear.

\section{Conclusion}

The one hit and two hit models have been formulated to account for the development of post-injury MOF in the absence of sepsis. The two hit model in particular is an attractive hypothesis that seems to account for the deterioration of patients in the intensive care unit brought about cumulatively by sequential insults. Additional credibility is provided by compelling laboratory evidence that inflammatory cells are indeed susceptible of priming and activation. A note of caution must be sounded, however, not to accept unquestioningly these inflammatory hypotheses. There is a large body of clinical work demonstrating an early postinjury production of cytokines that may lend some support to the one hit model. More attention should be paid specifically to those patients who develop early MOF. At present, there is a lack of data corroborating the two hit model. Before it is adopted, sound clinical studies need to establish a causal relationship firstly between intercurrent events and outburst of mediator production, and secondly between reactivation (or perpetuation) of inflammatory response and eventual MOF. Mediator, and particularly cytokine, release may have to be measured in tissues rather than in plasma. If the hypothesis is validated, the door might open to exciting prospects for pro- 
phylactic or therapeutic mediator blockade aimed at minimising or even counteracting the impact of second hits in surgical patients.

Conflict of interest: none.

Funding: none.

1 Tilney NL, Bailey GL, Morgan AP. Sequential system failure after rupture of abdominal aortic aneurysms: an unsolved problem in postoperative care. Ann Surg 1973;178:117-22.

2 Baue AE. Multiple, progressive or sequential systems failure: a syndrome of the 1970s. Arch Surg 1975;110:77981 .

3 Eiseman B, Beart R, Norton L. Multiple organ failure. Surg Gynecol Obstet 1977;144:323-6.

4 Fry DE, Pearlstein L, Fulton RL, et al. Multiple system organ failure: the role of uncontrolled infection. Arch Surg 1980;115:136-40

5 Faist E, Baue AE, Dittmer H, et al. Multiple organ failure in polytrauma patients. $\mathcal{F}$ Trauma 1983;23:775-82.

6 Goris RJA, Boekhorst TPAT, Nuytinck JKS, et al. Multiple organ failure: generalized autodestructive inflammation? Arch Surg 1985;120:1109-15.

7 Norton LW. Does drainage of intraabdominal pus reverse multiple organ failure ? Am f Surg 1985;149:347-50.

8 Saadia R, Schein M, MacFarlane C, et al. Gut barrier function and the surgeon. Br ₹ Surg 1990;77:487-92.

9 Saadia R. Trauma and bacterial translocation. Br 7 Surg 1995;82:1243-4.

10 American College of Chest Physicians/Society of Critical Care Medicine Consensus Conference. Definitions for sepsis and organ failure and guidelines for the use of innosepsis and organ failure and guidelines for the use of inno-
vative therapies in sepsis. Crit Care Med 1992;20:864-74.

11 Meakins JL. Etiology of multiple organ failure. $f$ Trauma 1990;30:S165-8.

12 Deitch EA. Multiple organ failure. Ann Surg 1992;216:11734

13 Moore FA, Moore EE. Evolving concepts in the pathogenesis of postinjury multiple organ failure. Surg Clin North Am 1995;75:257-77.

14 Biff WL, Moore EE. Splanchnic ischaemia/reperfusion and multiple organ failure. Br f Anaesth 1996;77:59-70.

15 Waydhas C, Nast-Kolb D, Jochum M, et al. Inflammatory mediators, infection, sepsis and multiple organ failure after severe trauma. Arch Surg 1992;127:460-7.

16 Sauaia A, Moore FA, Moore EE, et al. Pneumonia: cause or symptom of postinjury multiple organ failure? $\mathrm{Am} \mathcal{F} \mathrm{Surg}$ 1993;166:606-11.

17 Moore FA, Sauaia A, Moore EE, et al. Postinjury multiple organ failure: a bimodal phenomenon. $\mathcal{f}$ Trauma 1996;40: 501-12

18 Vercellotti GM, Yin HQ, Gustafson KS, et al. Plateletactivating factor primes neutrophil responses to agonists: role in promoting neutrophil-mediated endothelial damage. Blood 1988;71:1100-7.

19 Forehand JR, Pabst MJ, Phillips WA, et al. Lipopolysaccharide priming of human neutrophils for an enhanced respiratory burst: role of intracellular free calcium. $\mathcal{F}$ Clin Invest 1989;83:74-83.

20 Anderson BO, Harken AH. Multiple organ failure: inflammatory priming and activation sequences promote autologous tissue injury. F Trauma 1990;30:S44-9.

21 Brom J, Konig W. Cytokine-induced (interleukins-3, -6 and -8 , and tumour necrosis factor-beta) activation and deactivation of human neutrophils. Immunology 1992;75:281-5.

22 Mileski WJ, Winn RK, Harlan JMunology $1992,75: 281-5$. toxin in rabbits is increased after hemorrhagic shock. $\mathcal{F} A p p l$ toxin in rabbits is increased

23 Moore EE, Moore FA, Franciose RJ, et al. The postischemic gut serves as a priming bed for circulating neutrophils that gut serves as a priming bed for circulating neutrophils that
provoke multiple organ failure. $\mathcal{F}$ Trauma $1994 ; 37: 881-7$

24 Meldrum DR, Cleveland JC, Moore EE, et al. Adaptive and Meldrum DR, Cleveland JC, Moore EE, et al. Adaptive and
maladaptive mechanisms of cellular priming. Ann Surg maladaptive mech

25 Blackwell TS, Christman JW. Sepsis and cytokines: current status. Br F Anaesth 1996;77:110-7.

26 Partrick DA, Moore FA, Moore EE, et al. The inflammatory profile of interleukin-6, interleukin-8 and soluble intercellular adhesion molecule-1 in postinjury multiple organ failure. Am f Surg 1996;172:427-31.

27 Roumen RMH, Hendriks T, van der Ven-Jongekriig J, et al. Cytokine patterns in patients after major vascular surgery, hemorrhagic shock and severe blunt trauma. Relation with subsequent adult respiratory distress syndrome and multiple organ failure. Ann Surg 1993;218:769-76.

28 Hoch RC, Rodriguez R, Manning T, et al. Effects of accidental trauma on cytokine and endotoxin production. Crit Care Med 1993;21:839-45.

29 Sherry RM, Cue JI, Goddard JK, et al. Interleukin-10 is associated with the development of sepsis in trauma patients. F Trauma 1996;40:613-17.

30 Simons RK, Hoyt DB, Winchell RJ, et al. Elevated selectin levels after severe trauma: a marker for sepsis and organ failure and a potential target for immunomodulatory failure and a potential target fo

31 Schinkel C, Faist E, Zimmer S, et al. Kinetics of circulating adhesion molecules and chemokines after mechanical adhesion molecules and chemokines after

32 Svoboda P, Kantorová I, Ochmann J. Dynamics of interleukin 1,2 and 6 and tumor necrosis factor alpha in multiple trauma patients. $\mathcal{F}$ Trauma 1994;36:336-40.
33 Biffl WL, Moore EE, Moore FA, et al. Interleukin-6 in the injured patient. Marker of injury or mediator of inflammainjured patient. Marker of injury
tion? Ann Surg 1996;224:647-64.

34 Waydhas C, Nast-Kolb D, Trupka A, et al. Posttraumatic inflammatory response, secondary operations and late multiple organ failure. $\mathcal{F}$ Trauma 1996;40:624-31

35 Sautner T, Götzinger P, Redl-Wenzl EM, et al. Does reoperation for abdominal sepsis enhance the inflammatory host response? Arch Surg 1997;132:250-5.

36 Schein M, Wittmann DH, Holzheimer R, et al. Hypothesis: compartmentalization of cytokines in intraabdominal infection. Surgery 1996;119:694-700.

37 Grewal HP, Kotb M, El Din AM, et al. Induction of tumor necrosis factor in severe acute pancreatitis and its subsequent reduction after hepatic passage. Surgery 1994; 115:213-21.

38 Riché F, Dosquet C, Panis Y, et al. Levels of portal and systemic blood cytokines after colectomy in patients with carcinoma or Crohn's disease. $\mathcal{f}$ Am Coll Surg 1995;180:71824.

39 Rodriguez JL, Miller CG, Garner WL, et al. Correlation of the local and systemic cytokine response with clinical outthe local and systemic cytokine response with clinical out

come following thermal injury. F Trauma 1993;34:684-95. response in peritoneal exudate and plasma of patients undergoing planned relaparotomy for severe secondary peritonitis. Arch Surg 1995;130:1314-20.

\section{Commentary}

The article by Saadia and Schein on multiple organ failure (MOF) asks a fundamental question relating to its pathophysiology. In the late 1970 s about $20 \%$ of trauma deaths were described as occurring "late". Nearly all of these were the result of MOF in its various guises. ${ }^{1}$ Twenty years on the proportion of "late" deaths has not changed. ${ }^{2}$ MOF remains the main cause of "late" trauma deaths." Despite advances in intensive care and supportive treatment for failing organs the last 20 years have been marked by no discernible reduction in MOF mortality. ${ }^{56}$ The key to improving this situation, apart from reducing the number of accidents, is to reach a better understanding of the link between trauma and the subsequent development of organ failure, often many days later.

The "two hit" concept provides just such a link. In essence though, like many other ideas in the pathophysiology of trauma, it is not a new one. The Shwartzman reaction is the classic example of a two hit process. A rabbit is prepared by an intradermal injection of endotoxin. An intravenous injection of endotoxin, given after a latent period (usually 24 hours), then results in a haemorrhagic and necrotic lesion at the site of the initial inoculation. The second insult does not have to be endotoxin: relatively innocuous substances, such as agar and starch, will also provoke the reaction. An appreciation of this reaction led Jacob Fine's team to perform experiments in the $1950 \mathrm{~s}$, which showed that after haemorrhagic shock dogs were unable to resist a normally nonlethal dose of Escherichia coli. ${ }^{7}$ At the time neutrophil priming and activation were unknown concepts, but it was clear that for a 48 hour period after the haemorrhagic insult there was a deficiency in the host defence mechanism. A reduction in leucocyte mobilisation was postulated.

This type of experimental model has been revisited. ${ }^{8}$ One group of pigs received a 30 minute $E$ coli infusion two days after a four hour "Wigger's" type haemorrhagic insult and resuscitation. ${ }^{8}$ Pigs receiving this double insult showed greater reductions in blood pressure, 